\title{
A Survey of Quasar Clustering; Initial Results at the SGP*
}

\author{
M.J. Drinkwater \\ Institute of Astronomy \\ University of Cambridge \\ Cambridge CB3 OHA \\ England
}

\begin{abstract}
Several U.K.Schmidt objective prism plates are being searched for quasar clustering. The spectra are measured by the automated plate measuring facility (APM) and emission line objects selected for which redshifts can be estimated. Most redshifts tested (based on Lyman- $\alpha$ ) are accurate to $2 \%$. Initial results from the South Galactic Pole (SGP) field show little evidence of clustering on scales between 60 and $500 \mathrm{Mpc}\left(H_{0}=50, q_{0}=0\right.$, at $\left.z=2.3\right)$.
\end{abstract}

The use of automated techniques to find quasars has increased the surface density of candidates by more than an order of magnitude over that in visual surveys. The first plate in this survey (the SGP) has a

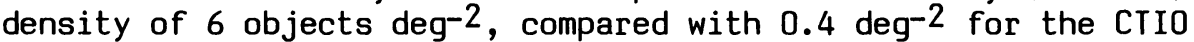
survey (Osmer 1981). The quasar candidates were selected from spectra on the U.K. Schmidt objective prism plate UJ3682 of the SGP, measured using the APM (see Hewett et al. 1985). To obtain the redshift estimates necessary for a clustering analysis, the candidate selection was based on the presence of emission lines with a signal-to-noise greater than 3 .

The redshift of each object was assigned by identifying the strongest emission feature with Lyman- $\alpha$. The APM spectrum was then inspected visually to confirm or alter this identification. This procedure was calibrated using 33 redshifts from Savage et al. (1985); 28 were correct to within $\Delta z= \pm 0.036$. (All the redshifts in the range $1.9<z<2.6$ were correctly identified so this range was used to define a clustering sample of 75 objects.) This accuracy is better than that claimed for objective prism data by Savage et al. (1985) and is near the resolution $(\Delta z= \pm 0.03)$ they require for clustering studies. In the above test the $1 \sigma$ redshift uncertainty was $\Delta z= \pm 0.02$, which corresponds to $38 \mathrm{Mpc}$ at $z=2.25$. This limits the tests of clustering to scales larger than about $60 \mathrm{Mpc}$. It was noted that the distribution of redshifts in the candidate list had an excess at $z=2.3$, although there was a significant number of objects at $z>2.5$. This reflects the greater ease of detecting Lyman- $\alpha$ emission compared to other lines, but it may also be caused by the inclusion of stars in the sample. In some stellar spectra the $4000 \mathrm{~A}$ break, if combined with strong G-band (4300A) absorption may resemble Lyman- $\alpha$ emission at $z=2.35$.

* Discussion on p.514 


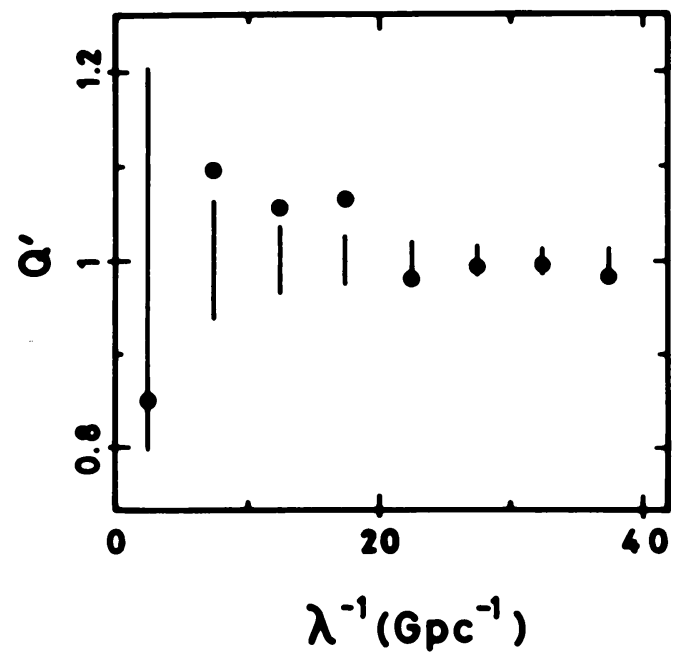

Figure 1. Histogram of $Q^{\prime}$ for the 3 dimensional PSA. The bars show the $\pm 1 \sigma$ range of expected values for a random distribution.

The sample of quasar candidates was tested for clustering using the power spectrum analysis (PSA) method of Webster (1976). The two dimensional results do not show evidence of clustering. The PSA results have a higher signal-to-noise when the test is applied in three dimensions. To do this the coordinates of each object were projected into a Euclidian box as in Webster (1982) using a Robinson-Walker metric with $H_{0}=50 \mathrm{kms}^{-1} / \mathrm{Mpc}$ and $q_{0}=0$ (see Osmer 1981). To allow for the non-uniform redshift distribution all terms in the $z$ direction were discarded in the calculation of $Q^{\prime}$. The resulting histogram in Figure 1 shows some evidence of clustering in the first four bins. The statistic $Q$, calculated under the hypothesis of clustering on a scale of $50 \mathrm{Mpc}$, was 1.065 , just outside the corresponding $3 \sigma$ range of $1.00 \pm 0.06$, although the degree of clustering is very weak. A close pair noted in the sample (1h3m11s, $-29^{\circ} 25^{\prime} 18^{\prime \prime}$ and $1 \mathrm{~h} 3 \mathrm{~m} 15 \mathrm{~s},-29^{\circ} 27^{\prime} 10^{\prime \prime}$ ' both at $z=2.25$; a separation of $12 \mathrm{Mpc}$ ) is not responsible for this result.

This initial study has established that it is possible to measure the clustering of quasars on scales between 60 and $500 \mathrm{Mpc}$ although there is little evidence for clustering in the SGP on such scales. The completion of the survey with about 10 plates (some 1000 objects in 224deg2) will increase the signal-to-noise by a factor of three making the detection of even very weak clustering possible.

\section{REFERENCES.}

Hewett,P.C., Irwin,M.J., Bunclark,P., Bridgeland,M.T., Kibblewhite,E.J., He,X.T., \& Smith,M.G., 1985. Mon. Not. R. astr. Soc., 213, 971.

Osmer,P.S., 1981. Astrophys. J., 247, 762.

Savage,A., Clowes, R.G., Cannon,R.D., Cheung,K., Smith,M.G., Boksenberg, A., \& Wall, J.V., 1985. Mon. Not. R. astr. Soc., 213, 485.

Webster,A.S., 1976. Mon. Not. R. astr. Soc., 175, 61.

Webster,A.S., 1982. Mon. Not. R. astr. Soc., 199, 683. 